\title{
Direct Measurement of Surface Diffusion Using Atom-Tracking Scanning Tunneling Microscopy
}

\author{
B. S. Swartzentruber \\ Surface and Interface Science, MS 1413, Sandia National Laboratories, Albuquerque, New Mexico 87185-1413
}

(Received 18 September 1995)

\begin{abstract}
The diffusion of $\mathrm{Si}$ dimers on the $\mathrm{Si}(001)$ surface at temperatures between room temperature and $128^{\circ} \mathrm{C}$ is measured using a novel atom-tracking technique that can resolve every diffusion event. The atom tracker employs lateral-positioning feedback to lock the scanning tunneling microscope (STM) probe tip into position above selected atoms with subangstrom precision. Once locked the STM tracks the position of the atoms as they migrate over the crystal surface. By tracking individual atoms directly, the ability of the instrument to measure dynamic events is increased by a factor of $\sim 1000$ over conventional STM imaging techniques.
\end{abstract}

PACS numbers: 68.35.Fx, 07.79.-v, 61.16.Ch, 68.10.Jy

The evolution of surface morphology during growth or etching depends on the detailed interplay between a myriad of atomic-scale kinetic processes. In order to control particular surface morphologies (e.g., to suppress or enhance overlayer island formation) it is important to achieve an understanding of the balance between thermodynamics and various kinetic processes. The equilibrium surface morphologies are controlled by thermodynamics, that is, the binding or configurational free energies, while the rates at which dynamic events occur on the surface are controlled by the details of the activation barriers - kinetics.

To study the evolution of surface morphology investigators often utilize rate-equation analysis [1], Monte Carlo [2], or molecular dynamics simulations [3] which explicitly require the input of specific atomic-scale energy parameters. The values for the input parameters are derived in a variety of ways ranging from first-principles total energy calculations, through semiempirical calculations, e.g., from the embedded-atom method, to purely empirical values chosen to reproduce particular measured morphological features. Of course, the ideal parameter values are derived from quantitative experimental measurements of particular system-specific atomic-scale processes. The quantitative measurements of individual atomic-scale energy parameters not only serve as inputs to realistic simulations and model calculations, but also enable the validation and refinement of such calculations. Such processes on certain metal systems have been studied for some time using field ion microscopy [4].

Because of its inherent atomic-scale resolution and access to a broad range of materials, the scanning tunneling microscope (STM) is ideally suited to studies of individual atomic-scale processes on surfaces, particularly since the advent of variable-temperature versions which enable the imaging of the surface as the rates of specific processes are varied by altering the sample temperature. However, conventional STM image acquisition is limited by the rate at which dynamic events can be resolved. In this work I report on the use of a novel atom-tracking technique that allows direct quantitative measurements of the diffusion of adsorbed silicon dimers on the $\mathrm{Si}(001)$ surface from room temperature to $128{ }^{\circ} \mathrm{C}$ over which the diffusion rate changes by 4 orders of magnitude. The groundwork for this investigation was laid in several recent studies of the structure and stability of silicon deposition on $\mathrm{Si}(001)$ in the low-coverage limit [5-7].

The experiments are performed in an ultrahigh vacuum chamber with a base pressure of $8 \times 10^{-11}$ torr that contains the STM and an evaporative silicon source. The clean surfaces are prepared by annealing the samples to $1250{ }^{\circ} \mathrm{C}$ for several seconds and then cooling to room temperature [8]. To evaporate silicon atoms onto the clean, cooled surface a nearby silicon wafer is heated to $\sim 1150^{\circ} \mathrm{C}$ and an intervening shutter is opened for $3-5 \mathrm{~s}$, resulting in the deposition of about $1 \%$ of a monolayer of silicon atoms.

When individual silicon atoms are deposited onto the surface at room temperature they diffuse rapidly and readily find another atom with which to form a stable cluster, i.e., an ad-dimer. As can be seen in Fig. 1(a) these ad-dimers appear as bright bumps in the STM images. The most energetically favorable location for the ad-dimers is to sit between the substrate dimer rows [5]. However, the majority of the ad-dimers formed on a room temperature substrate sit in metastable sites on top of the dimer rows, presumably due to the details of the kinetic pathway through which they are formed [5-7]. Examples of on-top and between-row ad-dimers are indicated in Fig. 1(a) by the solid and dashed circles, respectively. A barrier exists that impedes the on-top ad-dimers from falling off into the stable between-row sites.

The design of our microscope allows the repeated imaging of the surface at elevated temperatures enabling the measurement of atomic-scale dynamic events through the comparison of consecutive images [9,10]. Figures 1(a) and 1(b) show two images acquired at $65^{\circ} \mathrm{C}$ taken $29 \mathrm{~s}$ apart. The displacement of the ad-dimers along the top of the rows can be seen between the images [11]. An example 

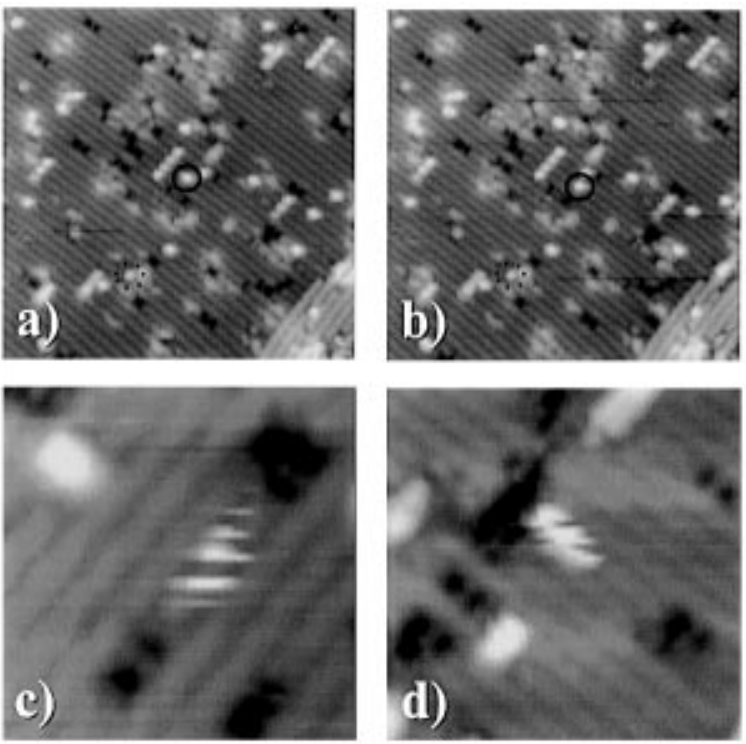

FIG. 1. High-temperature STM images of diffusing Si addimers. (a),(b) At $65^{\circ} \mathrm{C}$ diffusion is observed as displacements of the ad-dimers in sequential images (example in solid circle). Ad-dimers between the substrate rows are stable and do not diffuse (dashed circle). Scale $\sim 250 \AA$. (c) $\sim 70 \AA$ and (d) $95 \AA$ images taken at $128^{\circ} \mathrm{C}$ of two different ad-dimers trapped between substrate defects. These movie images can be viewed on the World Wide Web at http://www.sandia.gov/ surface_science/stm.

is circled in black. At this temperature the between-row ad-dimers are not observed to diffuse on the time scale of many minutes, nor do the diffusing on-top site dimers fall off into the stable between-row sites. The highly anisotropic diffusion of the on-top ad-dimers along the substrate dimer rows was previously studied at this temperature by Dijkkamp, van Loenen, and Elswijk [12].

In the earlier anneal and quench experiments in which samples were heated for a fixed amount of time and then cooled to room temperature before imaging, Zhang et al. [5] and Bedrossian [6] observed that after annealing the sample at $\sim 125{ }^{\circ} \mathrm{C}$ the barrier for an on-top site addimer to fall into the between-row sites is surmounted and on the time scale of several minutes the majority of ad-dimers are observed in the between-row sites. In images acquired at $128{ }^{\circ} \mathrm{C}$ for the present study, the vast majority of ad-dimers are also observed to be located in the stable between-row sites, or else in compact overlayer islands. Although most of the ad-dimers have found the stable binding sites, an occasional ad-dimer is observed that remains in the metastable sites on top of the dimer rows. Figures 1(c) and 1(d) show two images acquired at $128{ }^{\circ} \mathrm{C}$ of different ad-dimers diffusing on top of the substrate dimer rows. Each ad-dimer is trapped between two substrate defects that behave as repulsive hard walls. That these ad-dimers are observed and remain stable even after several hours at high temperature suggests that the simple transition from on-top site to between-row site has, in fact, a rather high barrier, and that a much lower barrier may exist that is mediated by surface defects or other neighboring clusters. The majority of the ad-dimers that are initially on top of the dimer rows may take this easy road to the minimum energy sites as they find neighboring clusters or defects, leaving a small percentage that are isolated from defects stuck in the higher energy sites.

The limitations of the conventional type of data acquisition are immediately apparent in Figs. 1(c) and 1(d). The images are acquired as a raster-scanned line by linestarting at the bottom of the image and moving to the top. For these specific $64 \times 64$ pixel images, the process takes $7.8 \mathrm{~s}$ to complete a frame, or $0.12 \mathrm{~s}$ per scan line. The diffusing ad-dimer is moving much more rapidly than the time scale of the data acquisition, and therefore its exact position cannot be defined within the images. The finite time response of image acquisition puts an upper bound on the temperature at which well-defined dynamic events can be measured-above this temperature the events are just occurring too rapidly. The primary cause of the inadequate time resolution in measuring, for example, the diffusion of the ad-dimers is the fact that during image acquisition the STM is spending most of the time not scanning the ad-dimer at all, but rather simply scanning the substrate away from the ad-dimer. Of course, the time scale can be decreased by zooming in and scanning a smaller area around the ad-dimer, however, this is limited because diffusion events quickly remove the ad-dimer from the field of view.

In order to quantitatively measure the diffusion kinetics of the ad-dimers, these limitations were overcome through the development of an atom-tracking technique in which the STM tip is locked onto a selected ad-dimer using twodimensional lateral feedback. Once locked, the feedback electronics maintain the tip over the cluster tracking its coordinates as it diffuses over the substrate. This procedure is accomplished by dithering the tip in a circle over the surface [13]. The quadrature output of a lock-in amplifier reading the tunnel current yields, independently, the $X$ and $Y$ local slopes of the surface, which in turn are the input error signals to the $X-Y$ lateral integrating feedback circuits [14]. Thus, this feedback maintains a lateral position of zero local slope-the top of the ad-dimer is tracked by continually "climbing uphill" [15]. In the atom-tracker mode, the STM spends all of its time measuring the kinetics of the selected addimer. When a diffusion event occurs the atom tracker quickly relocates to the ad-dimer's new position [16]. By utilizing atom tracking, the ability of the STM to resolve individual dynamic events is increased by about 3 orders of magnitude. Additionally, the measurement of every diffusion event eliminates the need to assume random walk statistics as is the case in mean square displacement measurements. In fact, the diffusion statistics can be measured explicitly. 
a)

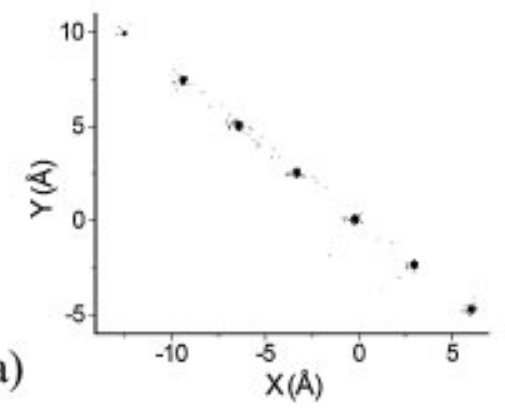

b)

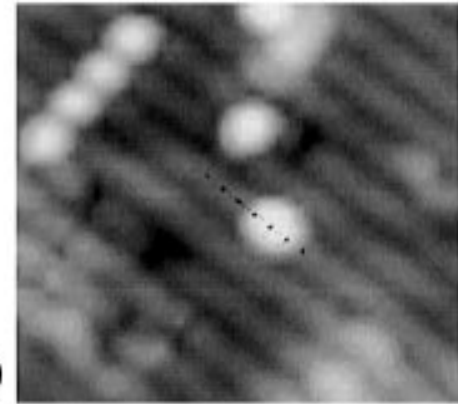

FIG. 2. (a) $X-Y$ scatter plot of data acquired at $65^{\circ} \mathrm{C}$. (b) Superposition of the data on the STM image taken immediately before atom tracking showing the initial location of the ad-dimer.

In STM measurements of kinetic processes, one must be wary of altering the surface dynamics through the presence of the probe, for it is well known that the interactions present in the tunnel junction between the probe tip and the sample can be used to physically manipulate adsorbed atoms and molecules on the surface [17]. This is a particular concern in the type of measurements presented here in which the tip is restricted to a specific sample region of interest. All of the data presented below were acquired with tunneling biases between -2.5 and $+2.5 \mathrm{~V}$ at $\leq 0.05 \mathrm{nA}$. Over this range of parameters, no variation of the diffusion kinetics of the ad-dimers could be detected.

The atom-track data consist of the $X, Y$, and $Z$ feedback coordinates as a function of time. Figure 2(a) shows a scatter plot of the complete set (11300 points) of lateral coordinates of the ad-dimer in the middle of Figs. 1(a) and 1(b) (circled in black) acquired at $65^{\circ} \mathrm{C}$ over $540 \mathrm{~s}$. During the course of the measurement the ad-dimer visited seven lattice sites along the top of the substrate dimer row, making 41 hops. The individual latticesite visitations are easily distinguishable in the scatter plot and are separated by the $\mathrm{Si}(001)$ lattice spacing of $3.84 \AA$. The data points at each lattice site group to $<1 \AA$, considerably smaller than the images would suggest, indicating how well the atom tracker locates the average position of the ad-dimer. This is particularly apparent when the scatter plot data are superposed on the topographic image taken immediately before atom tracking [Fig. 2(b)].

As described above, at $128^{\circ} \mathrm{C}$ the ad-dimers do not sit still long enough to be captured in a conventional image; however, the atom tracker easily locks on to them. In Fig. 1(d) there are eleven lattice sites between the hard-wall defect barriers on which the ad-dimer is confined to move. The amount of time that the addimer is observed on each lattice site is determined by the relative free energies of the sites. In a histogram of the site occupation probabilities (Fig. 3), it is clear that although the probability of finding the ad-dimer in one of the sites in the middle is approximately constant, the two end sites at the defects are occupied 2-3 times less frequently. The free energy of an ad-dimer on these two sites is $\sim 20-35 \mathrm{meV}$ higher than the others. A portion of this energy is probably attributable to the strain field in the lattice associated with the details of the defect structure. In this particular case only the end sites are appreciably affected. Other atomic configurations of the hard-wall defect barriers show different effects. In this context, the diffusing ad-dimer is a local probe of the atomic-scale substrate potential.

The frequency with which the ad-dimers hop is determined by the details of the activation barrier for diffusion. The hop frequency is measured by analyzing the ad-dimer coordinates as a function of time. Two of these time-line plots are shown at the top of Fig. 4 acquired at $65^{\circ} \mathrm{C}$ for $538 \mathrm{~s}$ and at $128^{\circ} \mathrm{C}$ for $22 \mathrm{~s}$, respectively. The mean residence time (time between hops) decreases from 13.1 to $0.11 \mathrm{~s}$ as temperature is increased. In the higher temperature data there are enough hops to extract a statistically significant residence-time probability distribution, shown at the bottom of Fig. 4 (taken from a data set with 736 hops over $79 \mathrm{~s}$ ). The dots are the measured values, and the solid line is an exponential with a decay constant equal to the measured mean residence time $\tau$. The quality of this comparison plot is indicative of a

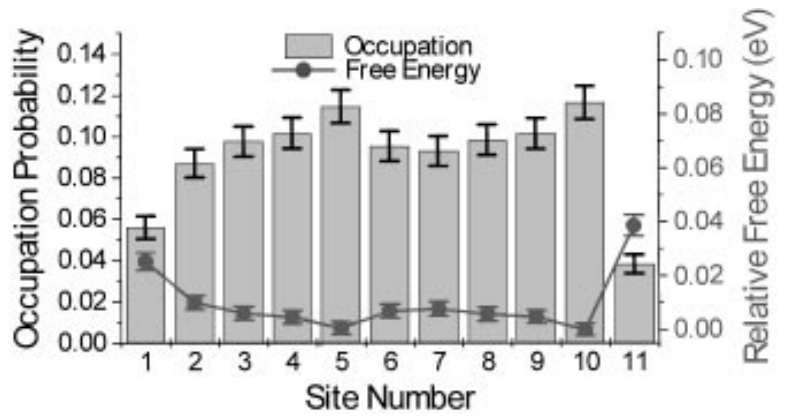

FIG. 3. Atom-track data taken at $128^{\circ} \mathrm{C}$. Site occupation probabilities and the extracted relative free energies of the eleven sites are measured from six data sets, total time is $196 \mathrm{~s}$. Error bars reflect the statistics related to the number of hops at each site. 


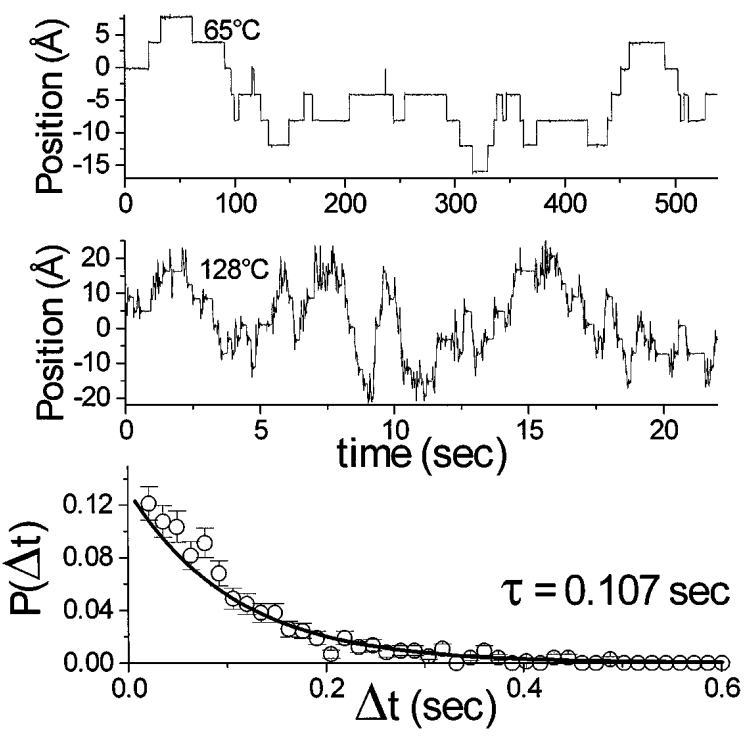

FIG. 4. Time-line plots. At the top are shown two plots of the ad-dimer position along the row as a function of time acquired at 65 and $128{ }^{\circ} \mathrm{C}$. The measured residence-time (time between hops) probability distribution at $128^{\circ} \mathrm{C}$ is shown at the bottom.

simply activated process, i.e., $1 / \tau=\nu_{0} \exp \left(-E_{a} / k T\right)$. The activation barrier $E_{a}$ is measured as the slope of the Arrhenius plot of the logarithm of the transition rate versus $1 / k T$. From the hopping rates measured at several points between 25 and $128^{\circ} \mathrm{C}$ (Fig. 5) the diffusion barrier for silicon ad-dimers on the $\mathrm{Si}(001)$ surface is determined to be $0.94 \pm 0.09 \mathrm{eV}$ [18]. This activation barrier for diffusion along the top of the substrate rows is higher than that measured for Si monomer diffusion, $0.7 \mathrm{eV}$ [19], although still substantially less than the barrier that must be overcome to remove atoms from the steps, $1.3 \mathrm{eV}$ [10]. The measured prefactor $\nu_{0}$ is $10^{12.8 \pm 1.3} \mathrm{~Hz}$. This value is similar to the generally accepted prefactor associated with measured metal-on-metal adatom diffusion for both simple hopping and concerted exchange displacement mechanisms [4].

The current work demonstrates the usefulness of atom tracking in performing quantitative measurements of the self-diffusion of $\mathrm{Si}$ ad-dimers on the $\mathrm{Si}(001)$ surface as a function of temperature by substantially increasing the range of hopping rates that can be resolved. By measuring every event, it is shown that the diffusion process is simply activated and that the binding energy can be locally affected by defects. In addition to employment in atomic-scale manipulation and lithography, the ability to lock onto and track surface dynamic events with the STM in real time and real space has a variety of additional important applications. Examples include studies of surface chemical processes such as oxidation and corrosion, atomic intermixing in heterogenous systems, electronic state evolution, as well as defect and adsorbate interactions at surfaces.

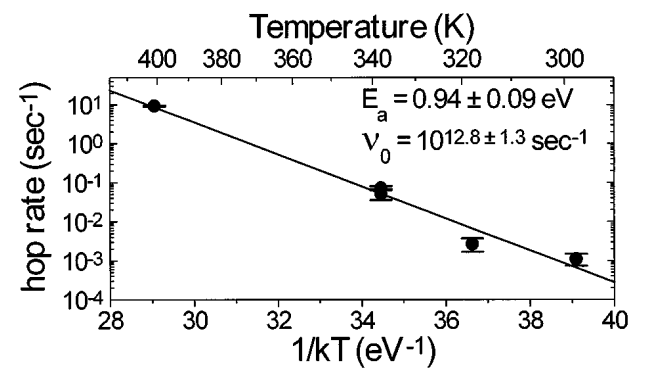

FIG. 5. Arrhenius plot of the logarithm of the diffusion rate versus $1 / k T$ from which the activation energy barrier is extracted.

This work performed at Sandia National Laboratories is supported by the U.S. Department of Energy under Contract No. DE-AC04-94AL85000.

[1] J. A. Venables, Surf. Sci. 299/300, 798 (1994).

[2] T. Kawamura, Prog. Surf. Sci. 44, 67 (1993).

[3] D. Srivastava and B. J. Garrison, Phys. Rev. B 47, 4464 (1993).

[4] G. L. Kellogg, Surf. Sci. Rep. 21, 1 (1994).

[5] Z. Zhang et al., Phys. Rev. Lett. 74, 3644 (1995).

[6] P. J. Bedrossian, Phys. Rev. Lett. 74, 3648 (1995).

[7] R. A. Wolkow, Phys. Rev. Lett. 74, 4448 (1995).

[8] B.S. Swartzentruber et al., J. Vac. Sci. Technol. A 8, 210 (1990).

[9] N. Kitamura et al., Phys. Rev. B 48, 5704 (1993).

[10] B. S. Swartzentruber and M. Schacht, Surf. Sci. 322, 83 (1995).

[11] Many of the substrate defects also change configuration between images, some of which involve a complicated rearrangement of many atoms.

[12] D. Dijkkamp, E. J. van Loenen, and H. B. Elswijk, in Ordering at Surfaces and Interfaces, edited by A. Yoshimori, T. Shinjo, and H. Watanabe (SpringerVerlag, Berlin, Heidelberg, 1992), p. 85.

[13] For the current work I used a dither circle diameter of $\sim 5 \AA$ at a frequency of $10 \mathrm{kHz}$. The dither amplitude must be large enough so that the convolution of the surface feature (ad-dimer) with the dither is larger than the hop length.

[14] D. W. Pohl and R. Möller, Rev. Sci. Instrum. 59, 840 (1988).

[15] Shifting the phase of the lock-in amplifier $180^{\circ}$ enables the atom tracker to lock onto depressions.

[16] The first-generation atom tracker employed in this work requires $<25 \mathrm{~ms}$ to relock onto an ad-dimer's new coordinate after a diffusion event.

[17] See, for example, J. A. Stroscio and D. M. Eigler, Science 254, 1319 (1991); Y.W. Mo, Science 261, 886 (1993); T.-C. Shen et al., Science 268, 1590 (1995).

[18] H.J.W. Zandvliet (unpublished) analyzed the squareddisplacement distribution of $\mathrm{Si}$ ad-dimers on $\mathrm{Si}(001)$ at $340 \mathrm{~K}(380 \mathrm{~K})$, and assuming a prefactor of $10^{13} \mathrm{~Hz}$ extracted an activation barrier of $1.02 \mathrm{eV}(1.06 \mathrm{eV})$.

[19] Y. W. Mo et al., Phys. Rev. Lett. 63, 2393 (1989). 